\title{
Novel Synthesis of Primary Arylamides from Aryl Methyl Ketone Oxidations using Iodine in Aqueous Ammonia
}

\author{
Norma A. Angeles, Felipe Villavicencio, Carlos Guadarrama, David Corona and \\ Erick Cuevas-Yañez*
}

Centro de Investigación en Química Sustentable UAEM-UNAM, Facultad de Química, Universidad Autónoma del Estado de México, Carretera Toluca-Atlacomulco Km 14.5 and Paseo Colón esq. Paseo Tollocan, 50120 Toluca, Mexico

\begin{abstract}
Primary arylamides were obtained when several aryl methyl ketones were treated with iodine in aqueous ammonia at room temperature in goods yields.

Arilamidas primárias foram obtidas em bons rendimentos quando diversas arilmetilcetonas foram tratadas com iodo em amônia aquosa a temperatura ambiente.
\end{abstract}

Keywords: amide, methyl ketone, iodine, ammonia, oxidation

\section{Introduction}

The amide group has a special importance in chemistry and biochemistry, since this functional group is the frame of several biological and pharmaceutical products and it is a point of departure en route to many natural products. ${ }^{1}$

Currently, one employed method to prepare aryl and heteroaryl amides involves the haloform cleavage reaction of trihalo acetyl derivatives. ${ }^{2-5}$ However, these procedures require anhydrous conditions and, in some cases, expensive reagents such as trichloroacetyl chloride for preparing the trichloroketone starting material. ${ }^{6-7}$ In addition, the use of water is preferred in environmental friendly procedures, which makes the chemical processes economical. ${ }^{8}$ These precedents motivated us to explore alternative routes to prepare amides from less expensive materials using mild conditions. In connection with other synthetic studies, we were attracted by the reports of Fang ${ }^{9-10}$ and Talukdar ${ }^{11}$ about the transformation of aldehydes to nitriles and amides, and we decided to apply these reactions in addition with other previous haloform reaction reports ${ }^{12-16}$ to the synthesis of primary amides from methyl ketones. This report describes our successful endeavors in this area.

In a model study, acetophenone was treated with an excess of iodine ( 3 molar equivalents) in aqueous ammonia and THF as cosolvent. After $1 \mathrm{~h}$, iodoform was separated and the product was extracted. Purification by crystallization afforded benzamide in $85 \%$ yield (Scheme 1).

In order to explore the reaction scope, several aryl and heteroaryl methyl ketones were reacted under similar conditions (see Table 1, Scheme 2). In general, the results showed that amides were the preferred products. It is noteworthy that ammonia concentration plays an important role, because the reaction is carried out only in a concentrated ammonium hydroxide solution, and at lower ammonia concentrations, the yields decreased or the amide is not formed, and only the starting material was isolated (Table 2).

The mechanism proposed for this reaction involves the triiodo methyl ketone formation, with the subsequent<smiles>CC(=O)c1ccccc1</smiles><smiles>ON=S</smiles><smiles>C[N+](C)=O</smiles>

Scheme 1. Reaction of acetophenone with iodine in aqueous concentrated ammonia.

*e-mail: ecuevasy@uaemex.mx 
<smiles>[R]C(C)=O</smiles>

Scheme 2. General reaction of methyl ketones with iodine in aqueous concentrated ammonia.

Table 1. Amides derived from diverse methyl ketones, iodine and ammonia

\begin{tabular}{|c|c|c|c|c|c|c|}
\hline Entry & Methyl ketone & Amide & Reaction time (h) & $\mathrm{mp}\left({ }^{\circ} \mathrm{C}\right)$ & mp lit. $\left({ }^{\circ} \mathrm{C}\right)$ & Yield (\%) \\
\hline 1 & acetophenone & benzamide & 1 & 127 & $128^{21}$ & 85 \\
\hline 2 & 4-methylacetophenone & 4-methylbenzamide & 1 & 137 & $137^{22}$ & 95 \\
\hline 3 & 2-methylacetophenone & 2-methylbenzamide & 1 & 140 & $141-142^{23}$ & 92 \\
\hline 4 & 4-chloroacetophenone & 4-chlorobenzamide & 0.5 & 175 & $172-176^{24}$ & 90 \\
\hline 5 & 4-fluoroacetophenone & 4-fluorobenzamide & 1 & 152 & $152-153^{24}$ & 94 \\
\hline 6 & 4-aminoacetophenone & 4-aminobenzamide & 24 & 181 & $181-183^{25}$ & 90 \\
\hline 7 & 4-methoxyacetophenone & 4-methoxybenzamide & 24 & 162 & $162-163^{26}$ & 83 \\
\hline 8 & 3-acetylthiophene & $\begin{array}{l}\text { thiophene-3-carboxylic } \\
\text { acid amide }\end{array}$ & 24 & 115 & - & 50 \\
\hline 9 & 1-methyl-3-acetylpyrrole & $\begin{array}{l}\text { 1-methylpyrrole-3-carboxylic } \\
\text { acid amide }\end{array}$ & 24 & 140 & - & 63 \\
\hline 10 & 2-acetylfuran & furamide & 12 & 142 & $142-143^{27}$ & 82 \\
\hline 11 & 2-acetylpyridine & pyridine-2-carboxylic acid amide & 12 & 109 & $108-109^{28}$ & 73 \\
\hline 12 & 3-acetylpyridine & pyridine-3-carboxylic acid amide & 12 & 129 & $128-131^{28}$ & 69 \\
\hline 13 & 4-acetylpyridine & pyridine-4-carboxylic acid amide & 12 & 157 & $157^{12}$ & 49 \\
\hline
\end{tabular}

Table 2. Effect of ammonia concentration in benzamide yield from acetophenone

\begin{tabular}{lcc}
\hline Ammonia & Reaction time (h) & Yield (\%) \\
Concentration (\%) & & \\
\hline 28 & 1 & 85 \\
25 & 3 & 75 \\
20 & 8 & 50 \\
15 & 24 & 0 \\
\hline
\end{tabular}

ammonia substitution (Scheme 3). Alternatively, enamine formation and subsequent iodination can also explain amide formation. At the moment, our group is investigating the mechanistic details of this reaction.<smiles>[R]C(C)=O</smiles><smiles>[R]C(=O)CI</smiles><smiles>[R]C(C)=N</smiles><smiles>[R]C(=C)CCCC(=N)CBr</smiles>

On the other hand, this process represents a new variant of the Haller-Bauer reaction, ${ }^{17-19}$ which allows the direct conversion of nonenolizable ketones to primary amides, with the advantage that strong bases such as sodium amide are not required.

\section{Conclusions}

In conclusion, the appropriately constituted methyl ketones are efficiently converted into primary amides through a simple and mild method. In addition, the procedure is economic and environmentally benign. These elements suggest that this route will enjoy widespread application.<smiles>[R]C(C)=O</smiles><smiles>[R]C(=N)N=C(C)CCCO</smiles>

Scheme 3. Mechanism of amide formation from methyl ketones and $\mathrm{I}_{2}-\mathrm{NH}_{3}$. 


\section{Experimental}

The starting materials were purchased from Aldrich Chemical Co. and were used without further purification. Solvents were distilled before use. Silica plates of $0.20 \mathrm{~mm}$ thickness were used for thin layer chromatography. Melting points were determined with a Fisher-Johns melting point apparatus and they are uncorrected. ${ }^{1} \mathrm{H}$ and ${ }^{13} \mathrm{C}$ NMR spectra were recorded using a Bruker AVANCE 300 , the chemical shifts $(\delta)$ are given in ppm relative to TMS as internal standard (0.00). For analytical purposes the mass spectra were recorded on a JEOL JMS-5X 10217 in the EI mode, $70 \mathrm{eV}, 200{ }^{\circ} \mathrm{C}$ via direct inlet probe. Only the molecular and parent ions $(\mathrm{m} / \mathrm{z})$ are reported. IR spectra were recorded on a Nicolet Magna 55-X FT instrument.

\section{Synthesis of amides from methyl ketones: CAUTION!}

Although we did not have any incidents by handling, it is known that iodine reacts with ammonia under certain conditions to generate nitrogen triiodide monoamine $\left(\mathrm{NI}_{3} \cdot \mathrm{NH}_{3}\right)$. The dry powder explodes readily by mechanical shock, heat or irradiation. ${ }^{20}$

\section{Typical procedure}

Iodine $(0.76 \mathrm{~g}, 3 \mathrm{mmol})$ was added to a solution of the appropriate ketone $(1 \mathrm{mmol})$ in $28 \%$ aqueous ammonia $(10 \mathrm{~mL})$ and THF $(1 \mathrm{~mL})$. The mixture was stirred for $1 \mathrm{~h}$ at room temperature. The dark solution became colorless and $10 \% \mathrm{Na}_{2} \mathrm{~S}_{2} \mathrm{O}_{3}(1 \mathrm{~mL})$ was added. Iodoform was filtered, the product was extracted with AcOEt $(3 \times 20 \mathrm{~mL})$. The organic phase was washed, dried over $\mathrm{Na}_{2} \mathrm{SO}_{4}$, and the solvent was evaporated in vacuo. The final product was purified by crystallization.

\section{Benzamide}

Yield 85\%, mp $127{ }^{\circ} \mathrm{C}(\mathrm{MeOH})$. IR $(\mathrm{KBr}) v_{\max } / \mathrm{cm}^{-1}$ : $3344,3162,1667 .{ }^{1} \mathrm{H}$ NMR $\left(300 \mathrm{MHz}, \mathrm{CDCl}_{3}\right) \delta 6.0$ (d, $2 \mathrm{H}), 7.47(\mathrm{~m}, 2 \mathrm{H}), 7.52(\mathrm{~m}, 1 \mathrm{H}), 7.82(\mathrm{~m}, 2 \mathrm{H}) .{ }^{13} \mathrm{C} \mathrm{NMR}$ $\left(75 \mathrm{MHz}, \mathrm{CDCl}_{3}\right) \delta 127.3,127.3,128.6,128.6,130.2$, 132.2, 169.0. MS [EI+] m/z (RI\%), $(\mathrm{M})^{+}(10), 120[\mathrm{M}-\mathrm{H}]^{+}$ (75), $105\left[\mathrm{M}-\mathrm{NH}_{2}\right]^{+}$(83), $77\left[\mathrm{M}-\mathrm{CONH}_{2}\right]^{+}(100)$.

\section{4-Methylbenzamide}

Yield 95\%, mp $137{ }^{\circ} \mathrm{C}(\mathrm{MeOH})$. IR (KBr) $v_{\max } / \mathrm{cm}^{-1}$ : $3343,3163,1667 .{ }^{1} \mathrm{H}$ NMR $\left(300 \mathrm{MHz}, \mathrm{CDCl}_{3}\right) \delta 2.40$ (s, $3 \mathrm{H}), 6.08$ (s, 2H), 7.22 (d, 2H, J 8.6 Hz), 7.69 (d, 2H, $J$ $8.2 \mathrm{~Hz}) .{ }^{13} \mathrm{C}$ NMR $\left(75 \mathrm{MHz}, \mathrm{CDCl}_{3}\right) \delta 21.4,127.3,127.3$, $129.2,129.2,130.9,142.5,169.4$. MS m/z (\%) $135[\mathrm{M}]^{+}$ (65), $119\left[\mathrm{M}-\mathrm{NH}_{2}\right]^{+}(100), 91\left[\mathrm{M}-\mathrm{CONH}_{2}\right]^{+}(65)$.

\section{2-Methylbenzamide}

Yield 92\%, mp $140{ }^{\circ} \mathrm{C}(\mathrm{MeOH})$. IR $(\mathrm{KBr}) v_{\max } / \mathrm{cm}^{-1}$ : 3343, 3163, 1667. ${ }^{1} \mathrm{H}$ NMR (300 MHz, $\left.\mathrm{CDCl}_{3}\right) \delta 2.48$ (s, $3 \mathrm{H}), 5.88(\mathrm{~s}, 1 \mathrm{H}), 6.27(\mathrm{~S}, 1 \mathrm{H})$ 7.24-7.46 (m, 4H). ${ }^{13} \mathrm{C}$ MNR $\left(75 \mathrm{MHz}, \mathrm{CDCl}_{3}\right) \delta 19.9,125.7,126.9,130.2,131.1,135.1$, 136.3, 171.2. MS m/z (\%) $135[\mathrm{M}]^{+}(65), 119\left[\mathrm{M}-\mathrm{NH}_{2}\right]^{+}$ (100), $91\left[\mathrm{M}-\mathrm{CONH}_{2}\right]^{+}$(77).

\section{4-Chlorobenzamide}

Yield $90 \%$, mp $175{ }^{\circ} \mathrm{C}(\mathrm{MeOH})$. IR $(\mathrm{KBr}) v_{\max } / \mathrm{cm}^{-1}$ : $3343,3162,1666 .{ }^{1} \mathrm{H}$ NMR $\left(300 \mathrm{MHz}, \mathrm{CDCl}_{3}\right) \delta 5.94$ (s, 2H) 7.41 (d, 2H, J 8.6 Hz), 7.95 (d, 2H, J 8.6 Hz). ${ }^{13} \mathrm{C} \mathrm{NMR}$ $\left(75 \mathrm{MHz}, \mathrm{CDCl}_{3}\right) \delta 128.9,128.9,129.3,129.3,132.5$, 138.0, 166.9. MS m/z (\%) $155[\mathrm{M}]^{+}(65), 139\left[\mathrm{M}-\mathrm{NH}_{2}\right]^{+}$ (100), $111\left[\mathrm{M}-\mathrm{CONH}_{2}\right]^{+}(45)$.

\section{4-Fluorobenzamide}

Yield $94 \%$, mp $152{ }^{\circ} \mathrm{C}\left(\mathrm{CH}_{2} \mathrm{Cl}_{2}\right)$. IR $(\mathrm{KBr}) v_{\max } / \mathrm{cm}^{-1}$ : 3326, 3150, 1640. ${ }^{~} \mathrm{H}$ NMR (300 MHz, $\left.\mathrm{CDCl}_{3}\right) \delta 5.94$ (s, $2 \mathrm{H}), 7.35(\mathrm{~d}, 2 \mathrm{H}, J 8.3 \mathrm{~Hz}), 7.92(\mathrm{~d}, 2 \mathrm{H}, J 8.3 \mathrm{~Hz}) .{ }^{13} \mathrm{C}$ $\operatorname{NMR}\left(75 \mathrm{MHz}, \mathrm{CDCl}_{3}\right) \delta 115.6,115.6,129.9,129.9,131.4$,

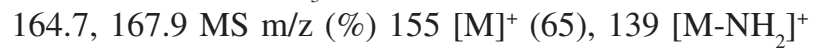
(100), $111\left[\mathrm{M}-\mathrm{CONH}_{2}\right]^{+}(45)$.

\section{4-Aminobenzamide}

Yield 90\%, mp $181{ }^{\circ} \mathrm{C}(\mathrm{MeOH})$. IR (KBr) $v_{\max } / \mathrm{cm}^{-1}$ : 3343, 3163, 1667. ${ }^{1} \mathrm{H}$ NMR (300 MHz, $\left.\mathrm{CDCl}_{3}\right) \delta 5.45$ (s, $2 \mathrm{H}), 6.52(\mathrm{~d}, 2 \mathrm{H}, J 8.76 \mathrm{~Hz}), 6.73(\mathrm{~s}, 1 \mathrm{H}), 7.44(\mathrm{~s}, 1 \mathrm{H})$, $7.58(\mathrm{~d}, 2 \mathrm{H}, J 8.76 \mathrm{~Hz}) .{ }^{13} \mathrm{C}$ NMR $\left(75 \mathrm{MHz}, \mathrm{CDCl}_{3}\right) \delta$ $112.4,112.4,120.9,128.9,128.9,151.4,168.1 . \mathrm{MS} \mathrm{m} / \mathrm{z}$ (\%) $136[\mathrm{M}]^{+}(80), 120\left[\mathrm{M}-\mathrm{NH}_{2}\right]^{+}(100), 92\left[\mathrm{M}-\mathrm{CONH}_{2}\right]^{+}$ (30).

\section{4-Methoxybenzamide}

Yield 83\%, mp $162{ }^{\circ} \mathrm{C}(\mathrm{MeOH})$. IR $(\mathrm{KBr}) \mathrm{v}_{\max } / \mathrm{cm}^{-1}$ : 3308, 3172, 1617. ${ }^{1} \mathrm{H}$ NMR $\left(300 \mathrm{MHz}, \mathrm{CDCl}_{3}\right) \delta 3.89$ (s, $3 \mathrm{H}), 6.98(\mathrm{~d}, 2 \mathrm{H}, J 8.7 \mathrm{~Hz}), 7.44(\mathrm{~s}, 2 \mathrm{H}), 7.90$ (d, 2H, $J$ $8.7 \mathrm{~Hz}) .{ }^{13} \mathrm{C}$ NMR $\left(75 \mathrm{MHz}, \mathrm{CDCl}_{3}\right) \delta 56.0,114.2,114.2$, 127.0, 129.9, 129.9, 163.4, 169.7. MS m/z (\%) $151[\mathrm{M}]^{+}$ (60), $135\left[\mathrm{M}-\mathrm{NH}_{2}\right]^{+}(100)$.

\section{Thiophene-3-carboxylic acid amide}

Yield 50\%, mp $115{ }^{\circ} \mathrm{C}(\mathrm{MeOH})$. IR (KBr) $v_{\max } / \mathrm{cm}^{-1}$ : 3343, 3163, 1667. ${ }^{1} \mathrm{H}$ NMR (300 MHz, $\left.\mathrm{CDCl}_{3}\right) \delta 7.33$ $\left(\mathrm{dd}, 1 \mathrm{H}, J_{l} 0.9 \mathrm{~Hz}, J_{2} 4.8 \mathrm{~Hz}\right), 7.53\left(\mathrm{dd}, 1 \mathrm{H}, J_{1} 0.9 \mathrm{~Hz}, J_{2}\right.$ $4.8 \mathrm{~Hz}), 8.10\left(\mathrm{dd}, 1 \mathrm{H}, J_{1} 0.9 \mathrm{~Hz}, J_{2} 2.7 \mathrm{~Hz}\right) .{ }^{13} \mathrm{C} \mathrm{NMR}(75$ $\left.\mathrm{MHz}, \mathrm{CDCl}_{3}\right) \delta 125.9,126.9,129.4,136.7,164.9 . \mathrm{MS} \mathrm{m} / \mathrm{z}$ (\%) $127[\mathrm{M}]^{+}(90), 111\left[\mathrm{M}-\mathrm{NH}_{2}\right]^{+}(100), 83\left[\mathrm{M}-\mathrm{CONH}_{2}\right]^{+}$ (30). HRMS (FAB ${ }^{+}$): for $\mathrm{C}_{6} \mathrm{H}_{9} \mathrm{NOS}$ Calc. 128.0170; Found 128.0173 . 
1-Methylpyrrole-3-carboxylic acid amide

Yield 63\%, mp $140{ }^{\circ} \mathrm{C}(\mathrm{MeOH})$. IR $(\mathrm{KBr}) v_{\max } / \mathrm{cm}^{-1}$ : $3342,3165,1668 .{ }^{1} \mathrm{H}$ NMR $\left(300 \mathrm{MHz}, \mathrm{CDCl}_{3}\right) \delta 3.68$ (s, $3 \mathrm{H}), 5.65(\mathrm{~s}, 2 \mathrm{H}), 6.57(\mathrm{~m}, 2 \mathrm{H}), 7.22(\mathrm{~m}, 1 \mathrm{H}) .{ }^{13} \mathrm{C}$ NMR $\left(75 \mathrm{MHz}, \mathrm{CDCl}_{3}\right) \delta 36.5,109.4,123.3,126.0,126.8$, 172.8. MS m/z (\%) $124[\mathrm{M}]^{+}(30), 108\left[\mathrm{M}-\mathrm{NH}_{2}\right]^{+}(100)$, $80\left[\mathrm{M}-\mathrm{CONH}_{2}\right]^{+}(20)$. HRMS $\left(\mathrm{FAB}^{+}\right)$: for $\mathrm{C}_{6} \mathrm{H}_{9} \mathrm{~N}_{2} \mathrm{O}$ Calc. 125.0175; Found 125.0177.

\section{Furamide}

Yield $82 \%$, mp $142{ }^{\circ} \mathrm{C}(\mathrm{MeOH})$. IR $(\mathrm{KBr}) \mathrm{v}_{\max } / \mathrm{cm}^{-1}$ : $3361,3123,1620 .{ }^{1} \mathrm{H}$ NMR $\left(300 \mathrm{MHz}, \mathrm{CDCl}_{3}\right) \delta 5.25$ (s, 2H), $6.52(\mathrm{~d}, 1 \mathrm{H}, J 3.6 \mathrm{~Hz}), 7.15(\mathrm{~d}, 1 \mathrm{H}, J 3.6 \mathrm{~Hz}), 7.60(\mathrm{~m}$, 1H). ${ }^{13} \mathrm{C}$ NMR $\left(75 \mathrm{MHz}, \mathrm{CDCl}_{3}\right) \delta 110.8,121.3,144.6$, 147.5, 162.2. MS m/z (\%) $111[\mathrm{M}]^{+}(80), 95\left[\mathrm{M}-\mathrm{NH}_{2}\right]^{+}(100)$.

\section{Pyridine-2-carboxylic acid amide}

Yield 72\%, mp $109{ }^{\circ} \mathrm{C}(\mathrm{MeOH})$. IR $(\mathrm{KBr}) \mathrm{v}_{\max } / \mathrm{cm}^{-1}$ : 3343, 3163, 1660. ${ }^{1} \mathrm{H}$ NMR (300 MHz, $\left.\mathrm{CDCl}_{3}\right) \delta 7.04$ (s, $1 \mathrm{H}), 7.45(\mathrm{~m}, 1 \mathrm{H}), 7.86(\mathrm{~m}, 1 \mathrm{H}), 8.00(\mathrm{~s}, 1 \mathrm{H}), 8.22(\mathrm{~m}, 1 \mathrm{H})$, $8.56(\mathrm{~m}, 1 \mathrm{H}) .{ }^{13} \mathrm{C}$ NMR $\left(75 \mathrm{MHz}, \mathrm{CDCl}_{3}\right) \delta 122.3,126.3$, 137.2, 148.2, 149.6, 167.3. MS m/z (\%): $122[\mathrm{M}]^{+}(30), 89$ $\left[\mathrm{M}-\mathrm{NH}_{2}\right]^{+}(100 \%), 92\left[\mathrm{M}-\mathrm{CONH}_{2}\right]^{+}(30 \%)$.

\section{Pyridine-3-carboxylic acid amide}

Yield 69\%, mp $129{ }^{\circ} \mathrm{C}(\mathrm{MeOH})$. IR $(\mathrm{KBr}) \mathrm{v}_{\max } / \mathrm{cm}^{-1}$ : 3343, 3163, 1660. ${ }^{1} \mathrm{H}$ NMR $\left(300 \mathrm{MHz}, \mathrm{CDCl}_{3}\right) \delta 7.04$ (s, $1 \mathrm{H}), 7.45(\mathrm{~m}, 1 \mathrm{H}), 8.07(\mathrm{~s}, 1 \mathrm{H}), 8.28(\mathrm{~m}, 1 \mathrm{H}), 8.75(\mathrm{~m}, 1 \mathrm{H})$, $9.18(\mathrm{~s}, 1 \mathrm{H}) .{ }^{13} \mathrm{C}$ NMR $\left(75 \mathrm{MHz}, \mathrm{CDCl}_{3}\right) \delta 122.4,128.8$, 134.8, 148.2, 151.0, 166.8. MS m/z (\%) $122[\mathrm{M}]^{+}(100)$, $106\left[\mathrm{M}-\mathrm{NH}_{2}\right]^{+}(60 \%), 78\left[\mathrm{M}-\mathrm{CONH}_{2}\right]^{+}(70 \%)$.

\section{Pyridine-4-carboxylic acid amide}

Yield 49\%, mp $157{ }^{\circ} \mathrm{C}(\mathrm{MeOH})$. IR $(\mathrm{KBr}) \mathrm{v}_{\max } / \mathrm{cm}^{-1}$ : 3343, 3161, 1667. ${ }^{1} \mathrm{H}$ NMR $\left(300 \mathrm{MHz}, \mathrm{CDCl}_{3}\right) \delta 7.22$ (s, $1 \mathrm{H}), 7.82(\mathrm{~d}, 2 \mathrm{H}, J 5.4 \mathrm{~Hz}), 8.12(\mathrm{~s}, 1 \mathrm{H}), 8.73(\mathrm{~d}, 2 \mathrm{H}, J$ $5.4 \mathrm{~Hz}) \cdot{ }^{13} \mathrm{C} \mathrm{NMR}\left(75 \mathrm{MHz}, \mathrm{CDCl}_{3}\right) \delta 121.1,121.1,140.7$, 149.6, 149.6, 167.0. MS m/z (\%) $122[\mathrm{M}]^{+}(100), 106[\mathrm{M}-$ $\left.\mathrm{NH}_{2}\right]^{+}(40 \%), 78\left[\mathrm{M}-\mathrm{CONH}_{2}\right]^{+}(55 \%)$.

\section{Acknowledgments}

Financial support from PROMEP-SEP is gratefully acknowledged. The authors would like to thank Rocío Patiño, Angeles Peña, Elizabeth Huerta, Javier Pérez and Luis Velasco (IQ-UNAM) for the technical support.

\section{References}

1. Reimann, J.E; Byerrum, R. U. In The Chemistry of Amides; Zabicky, J., Patai, S., eds.; Interscience Publishers: London, 1970, p. 601.
2. Hassinger, H. L.; Soll, R. M.; Gribble, G. W.; Tetrahedron Lett. 1998, 39, 3095.

3. Jiang, B.; Liu, J. F.; Zhao, S. Y.; J. Org. Chem. 2003, 68, 2376.

4. Kaiserman, J. A.; Gross, M.; Ge, Y.; White, S.; Hu, W.; Duan, J. X.; Baird, E. E.; Johnson, K. W.; Tanaka, R. D.; Moser, H. E.; Buerli, R. W.; J. Med. Chem. 2003, 46, 3914.

5. Mamidyala, S. K.; Firestine, S. M.; Tetrahedron Lett. 2006, 47, 7431.

6. Banwell, M. G.; Bray, A. M.; Willis, A. C.; Wong, D. A.; New J. Chem. 1999, 23, 687.

7. Bando, T.; Narita, A.; Saito, I.; Sugiyama, H.; J. Am. Chem. Soc. 2003, 125, 3471.

8. Lindström, U. M.; Chem. Rev. 2002, 102, 2751.

9. Shie, J. J.; Fang, J. M.; J. Org. Chem. 2007, 72, 3141.

10. Shie, J. J.; Fang, J. M.; J. Org. Chem. 2003, 68, 1158.

11. Talukdar, S.; Hsu, J. L.; Chou, T. C.; Fang, J. M.; Tetrahedron Lett. 2001, 42, 1103.

12. Jiménez, O.; Bosch, P.; Guerrero, A.; J. Org. Chem. 2005, 70, 10883.

13. Olivella S.; Solé A.; Jimenez O.; Bosch P.; Guerrero A.; J. Am. Chem. Soc. 2005, 127, 2620.

14. Levin D.; Kaszynski, P.; Mich, 1. J.; Organic Syntheses, Coll. Vol. X, 2004, 86 .

15. Zucco, C.; Lima, C. F.; Rezende, M. C.; Vianna, S. F.; Nome F.; J. Org. Chem. 1987, 52, 5356.

16. Staunton, J.; Eisenbraun, J.; Organic Syntheses, Coll. Vol. V, 1973; p. 8.

17. Ishihara, K.; Yano, T.; Org. Lett. 2004, 6, 1983.

18. Paquette, L. A.; Gilday, J. P.; Ra, S. C.; Hoppe, M.; J. Org. Chem. 1988, 53, 704.

19. Walborsky, H. M.; Allen, L. E.; Traenckner, H. J.; Powers, E. J.; J. Org. Chem. 1971, 36, 2937.

20. Roesky, H. W.; Möckel, K.; Chemical Curiosities, VCH: Weinheim, 1996, p. 292.

21. McMaster, L.; Langreck, F. B.; J. Am. Chem. Soc. 1917, 39, 103.

22. Feiring, A. E.; J. Org. Chem. 1976, 41,148.

23. Hartman, W. W.; Smith, L. A.; Organic Syntheses, Coll. Vol. II, 1943; p. 586.

24. Joshi, K. C.; Giri, S.; J. Indian Chem. Soc. 1960, 37, 423.

25. Prajapati, D.; Bora, H. N.; Sandhu, J. S.; Ghosh, A. C.; Synth. Commun. 1995, 25, 4025.

26. Chattopadhyaya, J. B.; Rao, A. V. R.; Tetrahedron 1974, 30, 2899.

27. Peng, Y.; Song, G.; Org. Prep. Proced. Int. 2002, 34, 95.

28. Sakai, K.; Ito, T.; Watanabe, K.; Bull. Chem. Soc. Jpn. 1967, 40,1660 . 